\title{
Apparent diffusion coefficient as an effective index for the therapeutic efficiency of brain chemoradiotherapy for brain metastases from lung cancer
}

\author{
Kai Liu, Zenglin Ma* and Lili Feng
}

\begin{abstract}
Background: The potential of apparent diffusion coefficient (ADC) value alteration before and after chemoradiotherapy as a potential monitor for therapeutic efficiency of treatment for brain metastases from lung cancer were discussed.

Method: Thirty lung cancer patients with brain metastases, conventional magnetic resonance imaging (MRI) examination and diffusion weighted imaging (DWI) were performed one week before chemoradiotherapy and after one treatment cycle and two treatment cycles. 43 tumor lesions were divided into effective group and invalid group according to the changes of the tumor size. The differences in ADC values at different time points before and after treatment in each treatment group were analyzed.

Result: The maximum diameter of the tumor was no difference after one treatment cycle, but decreased after two treatment cycles. ADC values significantly increased after both one and two treatment cycles. In effective group, the ADC values were significantly increased after one and two treatment cycles. While, there are no difference in invalid group after one treatment cycle but decreased after two treatment cycles. $\triangle A D C$ values in effective group after one and two treatment cycles were both significantly higher than those in the invalid group. ROC curve analysis then revealed that the area under the curve (AUC) of $\triangle A D C$ after one treatment was 0.872 .
\end{abstract}

Conclusion: ADC values in brain metastases from lung cancer can help monitor and dynamically observe the therapeutic efficiency of whole brain chemoradiotherapy.

Keywords: Apparent diffusion coefficient (ADC), Magnetic resonance imaging (MRI), Brain metastases, Therapeutic efficiency

\section{Background}

Brain metastases from are an increasingly common problem with an incidence ten times that of primary brain tumors and the diagnosis is often made by general radiologists $[1$, 2]. Cumulative incidence of brain metastases is noted to be particularly high for patients with locally advanced lung cancer and HER2 positive metastatic breast cancer, with figures of up to $30-60 \%$ reported [3-5].

Traditionally, magnetic resonance imaging (MRI) has been regarded as a gold standard for detection of brain

\footnotetext{
* Correspondence: zenglinma@163.com

Department of Radiology, The Third Affiliated Hospital of Beijing University of Chinese Medicine, No. 51 Xiaoguan Street, Andingmenwai, Chaoyang District, Beijing, People's Republic of China
}

metastases and used for assessing the site and number of metastases, planning for surgery or radiosurgery and assessing the response to therapy [6, 7]. However, the differentiation of high-grade gliomas from brain metastases represents a common differential diagnosis problem since both tumors may display similar imaging characteristics and contrast enhancement patters on conventional MRI [8]. Besides, it is not universally given to cancer patients who lack neurological symptoms at presentation. Even in patients with a normal MRI brain scan at diagnosis and limited-stage small cell lung cancer, a recent study found that $32.5 \%$ subsequently developed MRI evidence of brain metastases by the end of chemotherapy, before receiving prophylactic cranial irradiation [5]. In the course of radiotherapy for brain

(c) The Author(s). 2018 Open Access This article is distributed under the terms of the Creative Commons Attribution 4.0 International License (http://creativecommons.org/licenses/by/4.0/), which permits unrestricted use, distribution, and reproduction in any medium, provided you give appropriate credit to the original author(s) and the source, provide a link to the Creative Commons license, and indicate if changes were made. The Creative Commons Public Domain Dedication waiver (http://creativecommons.org/publicdomain/zero/1.0/) applies to the data made available in this article, unless otherwise stated. 
metastases from lung cancer, the changes of the tumor size exist significantly behind changes in biological and molecular levels. Thus, developing early biological indicators to measure radiotherapy efficacy has become an urgent need.

Newer MRI techniques have been applied to help diagnosis and prognosis. Diffusion-weighted magnetic resonance (MR) imaging detects the water mobility to reflect the morphologic and physiologic changes in tissues which was wildly applied in chest tumors characterization $[9,10]$. tThe apparent diffusion coefficient (ADC) of the lesion but these readings have more recently been suggested to have some prognostic value as well as diagnostic in brain metastases cancers $[11,12]$. An inverse relationship was observed between ADC values and tumor cellularity [8]. Previous studies had shown that ADC values can be used in differentiating between certain types of cerebral tumors [13-15]. However, the efficiency of the ADC values as an index of brain metastases, and its advantages over MRI remain controversial.

In the present study, we attempted to analyze the dynamic trends of maximal tumor diameter and ADC values at different time points after chemotherapy and whole-brain radiotherapy in patients with brain metastasis from lung cancer, to predict and to monitor the efficacy of $\triangle \mathrm{ADC}$ values as an index for brain metastasis from lung cancer after brain radiation therapy.

\section{Methods}

\section{Patients}

Institutional review board approval was obtained for this study. All the subjects were informed of the contents of the test and signed the informed consent voluntarily. From October 2013 to August 2016, 30 patients diagnosed with brain metastasis from lung cancer by pathology or clinical follow-up and clinical imaging tests were involved in the present study. The clinical characteristics of the 30 patients were shown in Table 1.

Case inclusion criteria: 1. Patients who were pathologically diagnosed with brain metastases from lung cancer, no limitation of gender and ethnicity; 2. Patients who were receiving radiotherapy and chemotherapy for the first time to ensure the uniformity of the initial state; 3. Patients with at least one measurable lesion (necrosis and cystic lesions not included) in the parenchyma of the brain, lesion diameter $>10 \mathrm{~mm}$.

Case exclusion criteria: 1. Patients who could not complete the entire treatment plan, the radiochemotherapy and Chinese medicine treatment were terminated in advance; 2 . Patients who received pacemaker implantation, coronary intervention, and Coronary Artery Bypass Grafting (CABG), thus contraindicated to MRI; 3. Patients who were unable to cooperate with MRI examinations.
Table 1 The clinical characteristics of enrolled patients

\begin{tabular}{ll}
\hline Clinical characteristics & Case number \\
\hline Lesion number & 43 \\
Mean age & 63.6 (45-85) \\
Gender & Female 18: Male 12 \\
Pathological type & 12 \\
Squamous carcinoma & 11 \\
Adenocarcinoma & 3 \\
Small cell lung cancer & 4 \\
Undifferentiated carcinoma & \\
Location of brain metastasis & 9 \\
Frontal lobe & 11 \\
Parietal lobe & 12 \\
Occipital lobe & 4 \\
Basal ganglia & 4 \\
Opisthencephalon & 2 \\
Temporal lobe & 1 \\
Brainstem &
\end{tabular}

\section{Treatment protocols}

Chemotherapy: oral administration of drugs that can pass through the blood-brain barrier, such as $\mathrm{CCNU}$ or me-CCNU, simultaneously supplemented by intramuscular injection of dexamethasone (MTX) once a week, 4 times as a treatment course, and the dehydration agent. Systemic chemotherapy is based on the patient's condition, the primary lesion and the cellular type of the cancer: for adenocarcinoma, CMF regimen (cyclophosphamide (CTX), MTX, fluorouracil (5-FU)) or CAF regimen (CTX, $\mathrm{M}$ (ADM), 5-FU] was used; for squamous carcinoma, DAV regimen (cisplatin (DDP), ADM, vincristine (VCR)) was used; for undifferentiated cancer, CVA regimen (CTX, VCR, ADM) was used. The above treatment was conducted once every 4 weeks, each time as a treatment cycle. Radiotherapy: Cobalt- 60 bilateral whole brain irradiation was conducted, the dosage was 4000-5000Gy, supplemented by mannitol dehydration or hormone therapy to reduce the radiotherapy response. Chinese traditional medicine: dialectical therapy.

\section{Examination protocols}

All examinations were performed on Achieva $1.5 \mathrm{~T} \mathrm{SE}$ MRI systems with an NV-8 combined coil for head and neck (Philips Healthcare, Amsterdam, Netherlands). The examinations were conducted with a gradient set of strength of $33 \mathrm{mT} / \mathrm{m}$ and a gradient switching rate of 122 $\mathrm{mT} / \mathrm{m} / \mathrm{ms}$. Conventional sequences, including T1-weighted images (T1WI), T2-weighted images (T2WI), DWI, FLAIR images, and transverse, coronal and sagittal T1WI contrast-enhanced MRI were obtained. MRI parameters are shown in Table 2. 
Table 2 Description of MRI parameters for Philips Achieva 1.5 T SE MRI systems

\begin{tabular}{llllll}
\hline & T1WI & T2WI & FLAIR & DWI & Contrast-enhanced $^{*}$ \\
\hline TR (ms) & 488 & 4748 & 8000 & 3090 & 156 \\
TE (ms) & 15 & 110 & 120 & 102 & 2.4 \\
TI (ms) & - & - & 2200 & - & - \\
Slice thickness (mm) & 6 & 6 & 6 & 6 & 6 \\
Slice gap (mm) & 1 & 1 & 1 & 1 & 1 \\
Slice number of scanning (layers) & 18 & 18 & 24 & 36 & 20 \\
Scanning time (s) & 24 & 42 & 2 & 1 & 22 \\
NSA & 1 & 2 & $230 \times 183$ & $230 \times 201$ & 2 \\
Field of view (mm²) & $230 \times 201$ & $230 \times 201$ & $256 \times 512$ & $152 \times 256$ & $388 \times 640$ \\
Matrix & $256 \times 256$ & $256 \times 256$ & 199 & 230 \\
\hline
\end{tabular}

*The Contrast-enhanced scan was performed by rapid bolus injection of gadopentate glucosamine (Gd-DTPA) at a dose of $0.1 \mathrm{mmol} / \mathrm{kg}$ through the cubital vein at a flow rate of $2.5 \mathrm{ml} / \mathrm{s}$. After bolus injection, $20 \mathrm{~mL}$ of saline was intravenously injected. B-values for DWl are $0 \mathrm{~s} / \mathrm{mm}^{2}$ and $1000 \mathrm{~s} / \mathrm{mm}^{2}$

\section{Image and data processing}

All measurements were performed on a Philips station. ADC maps were generated from the DWI sequence using Philips station. Image interpretation was done by two radiologists (five years of experience). The investigators were unaware of the patients' clinical and pathologic information.

The maximum diameter of the lesion was measured on the enhanced images combined with T2WI, T1WI, DWI and ADC images. In the tumor parenchyma region, the region of interest (ROI) was manually selected on the ADC map corresponding to the area of marked enhancement in the contrast-enhanced scan sequence. The ROI was placed in the lesion to select the region significantly enhanced in the contrast-enhanced scan sequence according to T1WI. Adjacent large blood vessels, cystic necrosis, and bleeding areas were avoided. Each lesion was measured three times, and ADC values were the average of 3 measurements. The cystic area after treatment should be included. ADC measurements were performed one week before comprehensive treatment, after one treatment cycle and two treatment cycles. The rates of $\mathrm{ADC}$ changes in effective and ineffective groups after one treatment cycle were calculated: $\triangle \mathrm{ADC}$ $=($ post-treatment ADC-pre-treatment $\mathrm{ADC}) /$ pre-treatment $\mathrm{ADC} \times 100 \%$. To measure the maximum diameter of the lesion, we measured the maximum cross-section of the lesion in the contrast-enhanced MRI and expressed it as a mean value of three measurements. If there were controversies between investigators, an average value for ADC was chosen by consensus.

\section{RECIST}

Patients with brain metastases underwent conventional MRI and contrast-enhanced MRI examinations at 1 week before treatment, one treatment cycle and two treatment cycles later. Treatment protocols are listed below. The layer with the maximum tumor diameter was selected; the maximum diameter or the maximum diameter summation of the tumor was measured and used as a grouping standard of the patients. Then the maximum diameters of the tumors 1 week before treatment and two treatment cycles later were compared. According to RECIST criteria, the evaluation of the target lesion is:

Complete remission (CR): the disappearance of almost all target lesions.

Partial remission (PR): the lesions were significantly reduced, and the summation of the maximum baseline diameter was reduced by $\geq 30 \%$.

Progression (PD): the summation of the maximum baseline diameter increased by $\geq 20 \%$ or new lesions appeared.

Stable (SD): the summation of the maximum baseline diameter reduced but did not reach PR or increased but did not reach PD.

The lesions that met with the CR and PR criteria were identified as effective groups, and the lesions that met the SD and PD criteria were classified as invalid groups.

\section{Statistical analysis}

The data was analyzed by SPSS20.0 software. The measurement data were expressed as mean \pm standard deviation (mean \pm S.D.). Normal distribution and homogeneity of variance tests were performed on different groups, and the data were in normal distribution. Paired $t$-test was used for comparison between paired samples. The ADC values pre-treatment and one treatment cycle after between the ineffective group and the effective group were compared using the independent sample $t$-test. The receiver operating characteristic (ROC) curve analysis was performed on the degree of change in the average ADC values of the patients treated for one cycle. $P<0.05$ indicates that the difference was statistically significant. 


\section{Result}

\section{ADC values indicate the tumor progression more} sensitively than MRI in brain metastases from lung cancer The maximum diameters of tumors and ADC values of brain metastases from lung cancer patients one week before treatment, one treatment cycle and two treatment cycles later were examined. The results showed that the maximum diameter of the tumor was no difference after one treatment cycle $(p=0.092)$; the maximum diameter of the tumor was significantly decreased after two treatment cycles $(p<0.001)$ (Fig. 1a, Table 3). However, ADC values increased significantly after one and two treatments cycles (Fig. 1b, Table 3), suggesting that ADC values indicate tumor progression more sensitively than the maximum diameters of tumors, especially after one treatment cycle.

\section{$\triangle A D C$ is a potential monitor of therapeutic efficacy on brain metastases from lung cancer}

Tumor lesions from patients were divided into two groups, effective group $(n=22)$ and invalid group (21), according to RECIST standard (details were shown in Fig. 2), and the ADC was examined. As Table 4 shown, before and after treatment cycles, the ADC values were no statistical difference between the effective group and invalid group. In the effective group, the $\mathrm{ADC}$ values were significantly increased after one and two treatment cycles. In the invalid group, the ADC values are not different after one treatment cycle, but decreased after two treatment cycles.

Then, $\triangle \mathrm{ADC}$ was calculated. As Table 5 shown, after one and two treatment cycles, $\triangle \mathrm{ADC}$ values in effective group were significantly higher than those in the invalid group. ROC curve analysis then revealed that the area under the curve (AUC) of $\triangle \mathrm{ADC}$ after one treatment cycle was 0.872 (sensitivity $=81.8 \%$, specificity $=85.7 \%$, 95\% CI: 0.805-0.992) (Fig. 3). These data indicating the potential of $\triangle \mathrm{ADC}$ as a novel index for brain metastasis progression and might help with monitoring therapeutic efficacy and distinguishing effective and invalid groups.

\section{Discussion}

Brain metastases from lung cancer are common intracranial tumors in adults and the incidence rate is about $20 \%$ [16]. With the continuous development of clinical examination methods, the increase in the incidence of lung cancer, and the prolongation of survival after radiotherapy and chemotherapy, the incidence of brain metastases from lung cancer has increased. In the past decades, the whole brain radiation therapy is still the standard treatment for brain metastasis from lung cancer [16], especially for lung cancer with multiple brain metastases.

Nowadays, clinicians commonly evaluate the therapeutic effect ofbrain metastasis from lung cancer by comparing the reduction of tumor size before and after treatment via CT or MRI [17]. Evaluating the therapeutic effect is critical to the further treatment of the patient. In the process of tumor treatment, establishing a method that can evaluate the treatment effect early and objectively becomes a considerable challenge. However, during the course of tumor treatment, the changes of tumor size exist significantly behind the changes in the biological and molecular levels $[18,19]$. Due to the different components of the tumor, it is unlikely that different types of tumors will
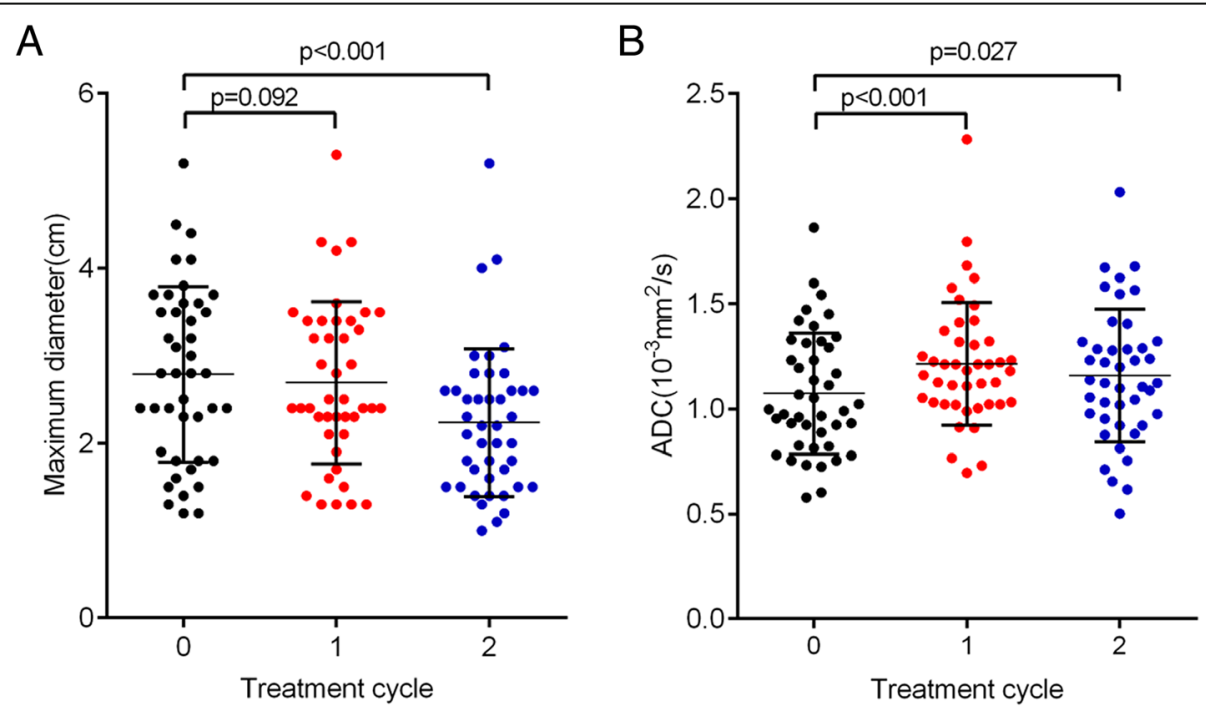

Fig. 1 ADC values indicate the tumor progression more sensitively than MRI (a) The maximum diameters of tumors for brain metastases from lung cancer before and after treatments. b ADC values of brain metastases from lung cancer before and after treatments. $n=43, p<0.05$ statistical significance compared to one week before treatment 
Table 3 The maximum diameters of tumors and ADC values for brain metastases from lung cancer $(n=43)$

\begin{tabular}{lll}
\hline Treatment & $\begin{array}{l}\text { The maximum } \\
\text { diameters of } \\
\text { tumors }(\mathrm{cm})\end{array}$ & $\begin{array}{l}\text { ADC values } \\
\left(10^{-3} \mathrm{~mm}^{2} / \mathrm{s}\right)\end{array}$ \\
\hline One week before treatment & $2.78 \pm 0.15$ & $1.07 \pm 0.29$ \\
One treatment cycle & $2.69 \pm 0.14$ & $1.21 \pm 0.29^{* *}$ \\
Two treatment cycles & $2.24 \pm 0.13^{* *}$ & $1.16 \pm 0.32^{*}$ \\
\hline
\end{tabular}

${ }^{*} p<0.05,{ }^{* *} p<0.01$, compared to one week before treatment

respond to the same treatment [20]. Unreasonable treatment may even accelerate the growth of tumors and causes tumor resistance. Developing the early measurement of biological indicators of therapeutic effectiveness has become an urgent need which can stop ineffective treatment in an early stage and reduce unnecessary toxicity and medical expenses.

Several imaging modalities were wildly applied in cancer diagnosis and assessment of therapy outcome. The tumor vascular physiology and hemodynamics could be measured by perfusion CT (PCT). The PCT parameters were considered as independent predictor of radiation therapy failure in head and neck cancer [21]. Diffusion tensor imaging (DTI) is valuable in diagnosing of idiopathic intracranial hypertension, differentiating gliomas grades and and residual head and neck cancer from post-radiation changes [22-24]. Moreover, combination with arterial spin labeling (ASL) perfusion increased the accuracy of MRI in distinguishing residual/recurrent gliomas from postradiation change [25]. DWI has changed the traditional diagnostic imaging model based on the anatomy and structural changes and led imaging diagnostics to a microscopic molecular level. It is currently the only non-invasive detection of water molecules free diffusion movement in vivo. The $\mathrm{ADC}$ value of the water molecules is determined by the viscosity of the molecules, the permeability of the cell membrane, the direction of the tissue, and the structure of the cells that impede the movement of the water molecules $[26,27]$. Therefore, the ADC values can distinguish tumor cells from non-cellular regions, cystic regions from solid regions, and the critical issues at the cellular level during tumor therapy. Recently, both animal and cell models have demonstrated that ADC values are of great importance in prognosis and the early monitoring of tumor therapeutic efficiency [28-30]. For example, lower ADC values were associated with greater tumor size and highly aggressive in cerebral cancers [31]. In addition, there are few human studies in this area, including gliomas [32], breast tumors [33], hepatic tumors [34], and rectal tumors [35], and all obtained the similar results.

Previous studies revealed that a higher ADC mean showed a longer overall survival regardless of adjuvant therapies in non-small cell lung cancer cerebral metastasis [36]. In the present study, we revealed that the significant increase of ADC values was appeared after one treatment cycle, which was earlier than the presence of

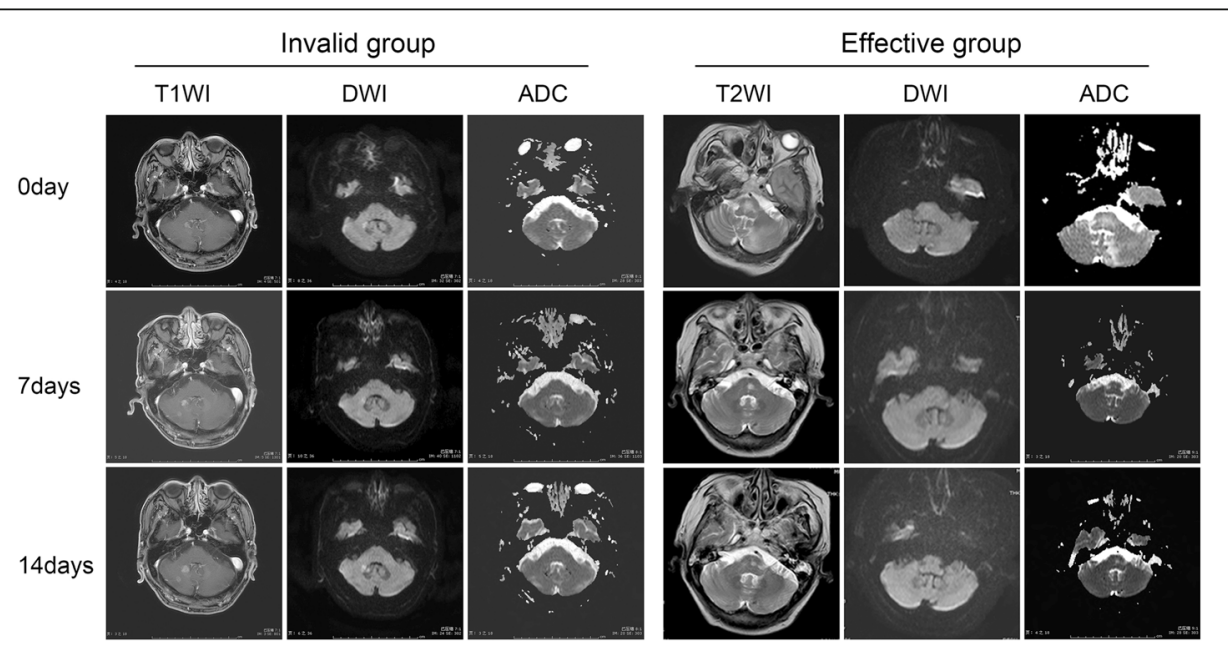

Fig. 2 Two cases were shown below for example the group division: Case 1: Pathologically diagnosed as brain metastases from right lung adenocarcinoma. The maximum diameter of the lesion was $10 \mathrm{~mm}$ one week before treatment and the ADC value was $0.653 \times 10^{-3} \mathrm{~mm} / \mathrm{s}$; the maximum diameter of the lesion was $12 \mathrm{~mm}$ and the ADC value was $0.733 \times 10^{-3} \mathrm{~mm}^{2} / \mathrm{s}$ after one treatment cycle; the maximum diameter of the lesion was $14 \mathrm{~mm}$ and the ADC value was $0.706 \times 10^{-3} \mathrm{~mm}^{2} / \mathrm{s}$ after two treatment cycles. According to RECIST criteria, the patient belonged to the invalid group. Case 2: pathologically diagnosed as brain metastases from lung cancer. The lesions on the right occipital lobe were nodular with slightly higher signal intensity on T2WI, slightly lower signal intensity on T1WI, high signal intensity on DWI, and obviously enhanced lesions on contrastenhanced T1WI. The maximum diameter was $17 \mathrm{~mm}$ before treatment and the ADC value was $0.759 \times 10^{-3} \mathrm{~mm}^{2} / \mathrm{s}$; after one treatment cycle, the maximum diameter of the lesion was reduced to about $16 \mathrm{~mm}$, and the ADC value was $1.05 \times 10^{-3} \mathrm{~mm}^{2} / \mathrm{s}$; after two treatment cycles, the enhanced part of the lesion was obviously reduced, with a diameter of $3 \mathrm{~mm}$, and the ADC value was $1.10 \times 10^{-3} \mathrm{~mm}^{2} / \mathrm{s}$, suggesting that the treatment was effective. According to RECIST criteria, this patient belongs to the effective group. 
Table 4 The change of ADC values after one and two treatment cycles in effective group and invalid group

\begin{tabular}{lll}
\hline Treatment & $\begin{array}{l}\text { ADC values of } \\
\text { effective group } \\
\left(10^{-3} \mathrm{~mm}^{2} / \mathrm{s}\right)\end{array}$ & $\begin{array}{l}\text { ADC values of } \\
\text { invalid group } \\
\left(10^{-3} \mathrm{~mm}^{2} / \mathrm{s}\right)\end{array}$ \\
\hline One week before treatment & $0.97 \pm 0.05$ & $1.17 \pm 0.06$ \\
One treatment cycle & $1.21 \pm 0.04^{* *}$ & $1.20 \pm 0.08$ \\
Two treatment cycles & $1.20 \pm 0.05^{* *}$ & $1.10 \pm 0.08^{*}$ \\
\hline
\end{tabular}

${ }^{*} p<0.05,{ }^{* *} p<0.01$, compared to before treatment, no difference of ADC value between effective group and invalid group under different treatment cycles

tumor maximum diameter alterations (after two treatment cycles). Effective anti-tumor therapy results in necrosis of tumor cells, reduced cell density, the disappearance of cell membrane integrity, increased extracellular space, and thus increased ADC values which can be sensitively detected by DWI [37]. Therefore, by observing the movement of water molecules in the tumor, the ADC value can be used as an early indicator to evaluate the effect of tumor treatment in brain metastasis from lung cancer.

Tumors with higher ADC values often contain more necrotic tissue and/or damaged cell membranes, with a poorer blood perfusion, and are relatively insensitive to radiotherapy [38], indicating that pre-radiotherapy ADC values can predict radiotherapy effects to a certain extent and can be used by clinicians to assess the sensitivity of tumors before radiotherapy. However, in the present study, no statistical difference was noted between effective group $\mathrm{ADC}$ values and invalid group $\mathrm{ADC}$ values before treatment cycle. In addition, we demonstrated that the $\triangle \mathrm{ADC}$ values were significantly higher in effective group than those in the invalid group. The AUC of $\triangle \mathrm{ADC}$ is 0.898 with $81.8 \%$ sensitivity and $85.7 \%$ specificity. These results demonstrated that $\triangle \mathrm{ADC}$ values have a potential to monitor therapeutic efficacy and distinguish chemoradiotherpy sensitive and insensitive brain metastasis from lung cancer.

There are a few limitations of this study. First, the small number of patients might limit the statistical results of the cases. Second, there is lack of long time follow-up of patients after treatment. The correlation of ADC with the overall survival in patients with brain metastasis from lung cancer is needed in the further studies.

Table 5 Comparison of $\triangle A D C$ values in effective group and invalid group

\begin{tabular}{lll}
\hline Treatment & $\begin{array}{l}\triangle A D C \text { values of } \\
\text { effective group (\%) }\end{array}$ & $\begin{array}{l}\triangle \text { ADC values of } \\
\text { invalid group (\%) }\end{array}$ \\
\hline One treatment cycle & $28.23 \pm 3.97^{*}$ & $2.5 \pm 2.27$ \\
Two treatment cycle & $27.56 \pm 5.16^{*}$ & $-7.44 \pm 2.62$ \\
\hline
\end{tabular}

${ }^{*} p<0.05$, compared to invalid group

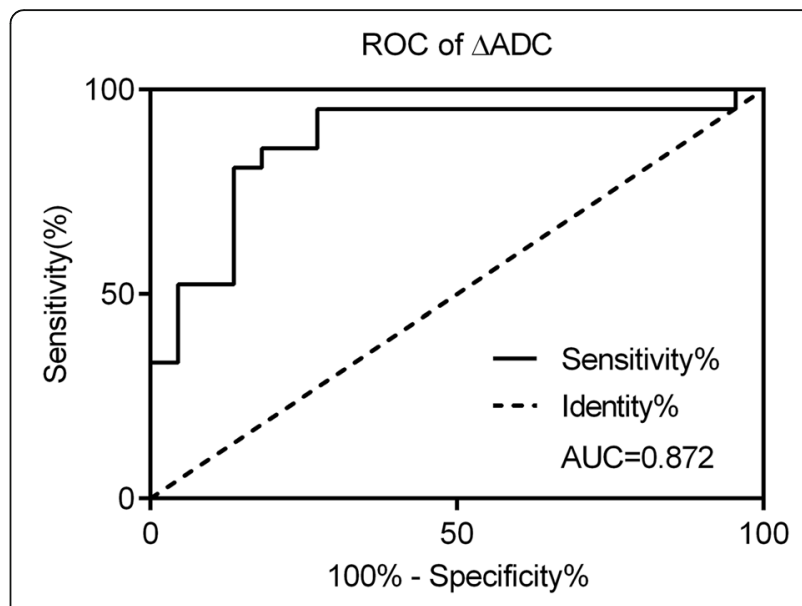

Fig. 3 ROC curve analysis of $\triangle A D C$ values after one treatment cycle. The AUC was 0.872 (sensitivity $=81.8 \%$, specificity $=85.7 \%$ )

\section{Conclusions}

In summary, $\mathrm{ADC}$ value examination during the chemoraidotherapy treatment cycles can help early monitoring and dynamic observation of therapeutic efficacy in brain metastases from lung cancer. It is possible for clinicians to quickly, accurately and non-invasively predict and monitor the responses of tumors before and during treatment, to stop ineffective treatment early and to reduce unnecessary toxicity and medical expenses.

\section{Abbreviations}

ADC: Apparent diffusion coefficient; ASL: Arterial spin labeling; AUC: The area under the curve; CABG: Coronary Artery Bypass Grafting; CR: Complete remission; DTI: Diffusion tensor imaging; DWl: Diffusion weighted imaging; PCT: Perfusion CT; PR: Partial remission; ROC: The receiver operating characteristic; T1WI: T1-weighted images; T2WI: T2-weighted images

\section{Funding}

The study was supported by Independent topic selection of Beijing University of Chinese Medicine in 2016 (Young and Middle-aged Teachers Project),No. 2016-JYB-JSMS-066.

Availability of data and materials

All data generated or analyzed during this study are included in this published article. The datasets used and/or analyzed during the current study are available from the corresponding author on reasonable request.

\section{Authors' contributions}

$\mathrm{KL}$ the mainly writer of the article, conceived and designed the experiments. LF Data analysis and statistical analysis. ZM Experimental guidance, conceived and designed the experiments, data verification. All authors have read and approved the manuscript.

\section{Ethics approval and consent to participate}

The study was conducted in accordance with the Declaration of Helsinki, and the protocol was approved by the Ethic Committee of The Third Affiliated Hospital of Beijing University of Chinese Medicine. All of the enrolled patients signed informed consent forms.

Consent for publication

Not applicable.

Competing interests

The authors declare that they have no competing interest. 


\section{Publisher's Note}

Springer Nature remains neutral with regard to jurisdictional claims in published maps and institutional affiliations.

Received: 16 July 2018 Accepted: 7 September 2018 Published online: 17 September 2018

\section{References}

1. Jenkinson MD, Haylock B, Shenoy A, Husband D, Javadpour M. Management of cerebral metastasis: evidence-based approach for surgery, stereotactic radiosurgery and radiotherapy. Eur J Cancer. 2011:47(5):649-55.

2. Nussbaum ES, Djalilian HR, Cho KH, Hall WA. Brain metastases. Histology, multiplicity, surgery, and survival. Cancer. 1996;78(8):1781-8.

3. Auperin A, Arriagada R, Pignon JP, Le Pechoux C, Gregor A, Stephens RJ Kristjansen PE, Johnson BE, Ueoka H, Wagner H, et al. Prophylactic cranial irradiation for patients with small-cell lung cancer in complete remission. Prophylactic cranial irradiation overview collaborative group. N Engl J Med. 1999:341(7):476-84.

4. Gore EM, Bae K, Wong SJ, Sun A, Bonner JA, Schild SE, Gaspar LE, Bogart JA, Werner-Wasik M, Choy H. Phase III comparison of prophylactic cranial irradiation versus observation in patients with locally advanced non-smallcell lung cancer: primary analysis of radiation therapy oncology group study RTOG 0214. J Clin Oncol. 2011;29(3):272-8.

5. Brufsky AM, Mayer M, Rugo HS, Kaufman PA, Tan-Chiu E, Tripathy D, Tudor IC, Wang LI, Brammer MG, Shing M, et al. Central nervous system metastases in patients with HER2-positive metastatic breast cancer: incidence, treatment, and survival in patients from registHER. Clin Cancer Res. 2011;17(14):4834-43.

6. Fink KR, Fink JR. Imaging of brain metastases. Surg Neurol Int. 2013;4(Supp 4):S209-19.

7. Young RJ, Sills AK, Brem S, Knopp EA: Neuroimaging of metastatic brain disease. Neurosurgery 2005, 57(5 Suppl):S10-S23; discusssion S11-14.

8. Chen L, Liu M, Bao J, Xia Y, Zhang J, Zhang L, Huang X, Wang J. The correlation between apparent diffusion coefficient and tumor cellularity in patients: a meta-analysis. PLoS One. 2013:8(11):e79008.

9. Razek AA. Diffusion magnetic resonance imaging of chest tumors. Cancer Imaging. 2012;12(3):452.

10. Abdel Razek AA, Gaballa G, Denewer A, Tawakol I. Diffusion weighted MR imaging of the breast. Acad Radiol. 2010;17(3):382-6.

11. Kim YJ, Chang KH, Song IC, Kim HD, Seong SO, Kim YH, Han MH. Brain abscess and necrotic or cystic brain tumor: discrimination with signal intensity on diffusion-weighted MR imaging. AJR Am J Roentgenol. 1998; 171(6):1487-90.

12. Berghoff AS, Spanberger T, Ilhan-Mutlu A, Magerle M, Hutterer M, Woehrer A, Hackl M, Widhalm G, Dieckmann K, Marosi C, et al. Preoperative diffusionweighted imaging of single brain metastases correlates with patient survival times. PLoS One. 2013;8(2):e55464.

13. Surov A, Ginat DT, Sanverdi E, Lim CC, Hakyemez B, Yogi A, Cabada T, Wienke A. Use of diffusion weighted imaging in differentiating between Maligant and benign Meningiomas. A multicenter analysis. World Neurosurg. 2016;88:598-602.

14. Kono K, Inoue Y, Nakayama K, Shakudo M, Morino M, Ohata K, Wakasa K, Yamada $R$. The role of diffusion-weighted imaging in patients with brain tumors. AJNR Am J Neuroradiol. 2001:22(6):1081-8.

15. Svolos P, Tsolaki E, Kapsalaki E, Theodorou K, Fountas K, Fezoulidis I, Tsougos I. Investigating brain tumor differentiation with diffusion and perfusion metrics at 3T MRI using pattern recognition techniques. Magn Reson Imaging. 2013;31(9):1567-77.

16. Kawabe T, Phi JH, Yamamoto M, Kim DG, Barfod BE, Urakawa Y. Treatment of brain metastasis from lung cancer. Prog Neurol Surg. 2012;25:148-55.

17. Binder D, Temmesfeld-Wollbruck B, Wurm R, Woiciechowsky C, Schaper C, Schurmann D, Suttorp N, Beinert T. brain metastases of lung cancer. Dtsch Med Wochenschr. 2006;131(4):165-71.

18. Pickles MD, Gibbs P, Lowry M, Turnbull LW. Diffusion changes precede size reduction in neoadjuvant treatment of breast cancer. Magn Reson Imaging. 2006;24(7):843-7.

19. Koh DM, Padhani AR. Diffusion-weighted MRI: a new functional clinical technique for tumour imaging. Br J Radiol. 2006;79(944):633-5.

20. Heppner GH, Miller FR. The cellular basis of tumor progression. Int Rev Cytol. 1998;177:1-56
21. Razek AA, Tawfik AM, Elsorogy LG, Soliman NY. Perfusion CT of head and neck cancer. Eur J Radiol. 2014:83(3):537-44.

22. El-Serougy L, Abdel Razek AA, Ezzat A, Eldawoody H, El-Morsy A. Assessment of diffusion tensor imaging metrics in differentiating low-grade from high-grade gliomas. Neuroradiol J. 2016;29(5):400.

23. Razek AAKA, Batouty N, Fathy W, Bassiouny R. Diffusion tensor imaging of the optic disc in idiopathic intracranial hypertension. Neuroradiology. 2018:1-8.

24. Razek AAKA. Diffusion tensor imaging in differentiation of residual head and neck squamous cell carcinoma from post-radiation changes. Magn Reson Imaging. 2018.

25. Razek AAKA, El-Serougy L, Abdelsalam M, Gaballa G, Talaat M. Differentiation of residual/recurrent gliomas from postradiation necrosis with arterial spin labeling and diffusion tensor magnetic resonance imaging-derived metrics. Neuroradiology. 2018;60(2):169-77.

26. Razek AAKA. Routine and advanced diffusion imaging modules of the salivary glands. Neuroimaging Clin N Am. 2018;28(2):245-54.

27. Kauppinen RA. Monitoring cytotoxic tumour treatment response by diffusion magnetic resonance imaging and proton spectroscopy. NMR Biomed. 2002;15(1):6-17.

28. Moffat BA, Hall DE, Stojanovska J, McConville PJ, Moody JB, Chenevert TL, Rehemtulla A, Ross BD. Diffusion imaging for evaluation of tumor therapies in preclinical animal models. MAGMA. 2004;17(3-6):249-59.

29. Jennings D, Hatton BN, Guo J, Galons JP, Trouard TP, Raghunand N, Marshall J, Gillies RJ. Early response of prostate carcinoma xenografts to docetaxe chemotherapy monitored with diffusion MRI. Neoplasia. 2002;4(3):255-62.

30. Galons JP, Altbach MI, Paine-Murrieta GD, Taylor CW, Gillies RJ. Early increases in breast tumor xenograft water mobility in response to paclitaxel therapy detected by non-invasive diffusion magnetic resonance imaging. Neoplasia. 1999;1(2):113-7.

31. Razek AA, Nada N. Correlation of choline/Creatine and apparent diffusion coefficient values with the prognostic parameters of head and neck squamous cell carcinoma. NMR Biomed. 2016;29(4):483-9.

32. Hamstra DA, Chenevert TL, Moffat BA, Johnson TD, Meyer CR, Mukherii SK, Quint DJ, Gebarski SS, Fan X, Tsien Cl, et al. Evaluation of the functional diffusion map as an early biomarker of time-to-progression and overall survival in high-grade glioma. Proc Natl Acad Sci U S A. 2005:102(46):16759-64.

33. Buijs M, Kamel IR, Vossen JA, Georgiades CS, Hong K, Geschwind JF. Assessment of metastatic breast cancer response to chemoembolization with contrast agent enhanced and diffusion-weighted MR imaging. J Vasc Interv Radiol. 2007;18(8):957-63.

34. Cui Y, Zhang XP, Sun YS, Tang L, Shen L. Apparent diffusion coefficient: potential imaging biomarker for prediction and early detection of response to chemotherapy in hepatic metastases. Radiology. 2008;248(3):894-900.

35. DeVries AF, Kremser C, Hein PA, Griebel J, Krezcy A, Ofner D, Pfeiffer KP, Lukas $P$, Judmaier $W$. Tumor microcirculation and diffusion predict therapy outcome for primary rectal carcinoma. Int J Radiat Oncol Biol Phys. 2003; 56(4):958-65.

36. Zakaria R, Das K, Radon M, Bhojak M, Rudland PR, Sluming V, Jenkinson MD. Diffusion-weighted MRI characteristics of the cerebral metastasis to brain boundary predicts patient outcomes. BMC Med Imaging. 2014; $14(1): 26$.

37. Lee KC, Moffat BA, Schott AF, Layman R, Ellingworth S, Juliar R, Khan AP, Helvie M, Meyer CR, Chenevert TL, et al. Prospective early response imaging biomarker for neoadjuvant breast cancer chemotherapy. Clin Cancer Res. 2007;13(2 Pt 1):443-50

38. Koh DM, Scurr E, Collins D, Kanber B, Norman A, Leach MO, Husband JE. Predicting response of colorectal hepatic metastasis: value of pretreatmen apparent diffusion coefficients. AJR Am J Roentgenol. 2007:188(4):1001-8. 